\title{
Mechanical properties of mammalian single smooth muscle cells \\ I. A low cost large range microforce transducer
}

\author{
J. J. GLERUM* and R. VAN MASTRIGT, \\ Departments of Urology and Biomedical Physics and Technology, Erasmus University, Rotterdam, The Netherlands.
}

Received 27 June 1989; accepted 18 April 1990

\section{Summary}

A transducer has been developed for measuring the minute forces generated during isometric contractions $(1.0-10.0 \mu \mathrm{N})$ of single smooth muscle cells from the pig urinary bladder and the human uterus.

In addition to its high sensitivity, resolution and stability ( $100 \mathrm{mV} \mu \mathrm{N}^{-1},<0.1 \mu \mathrm{N}$ and $<2.0 \mu \mathrm{N} \mathrm{h}^{-1}$ ), the transducer features a very wide range (100-140 $\mu \mathrm{N})$ with good linearity, enabling measurement of contractions as well as passive force-length characteristics within one uninterrupted measurement session.

Since the transducer features an independent and interchangeable force to displacement conversion system, different force ranges can be realized by inserting force conversion systems with different compliances.

\section{Introduction}

To measure passive force-length characteristics of single smooth muscle cells from pig urinary bladders or human uteruses over the whole length range of these cells, $100-200 \mu \mathrm{m}$, one needs a force transducer with: (1) a large linear range; (2) a small compliance; (3) a longitudinal pull on the cell; (4) a horizontal working axis (so as to keep the cell in focus while stretching more and more) and (5) a good long term stability. For contractility measurements, the transducer should also: (6) have a high sensitivity; (7) show a good short term stability, and (8) be insensitive to electrical currents or fields (resulting from electrical stimulation) and, as mammalian cells are to be investigated at $37^{\circ} \mathrm{C}$, it should, (9) be insensitive to external heat sources. Because measurements are conducted under a microscope, the transducer should (10) not be sensitive to external light sources, and (11) be sufficiently small to fit easily on the object stage without occupying to much space necessary for other instruments and manipulators. For electrophysiological measurements on the same cell, (12) the transducer should not emit any stray magnetic fields or (13) cause electric currents in the incubation bath. To avoid unnecessary loss of valuable experimentation time the transducer should be (14) repairable instantaneously at low cost.

A considerable number of papers on ultra sensitive force transducers have recently been published (Meiss, 1971, 1974; Minns \& Franz, 1972; Canaday \& Fay 1976; etc). The most commonly known designs are the transducers according to Canaday, Warshaw and Fay (Canaday \& Fay, 1976; Warshaw \& Fay, 1983), and the variations to the design of Meiss (1971, 1974). Other transducer types were published by Ishii (Ishii \& Takahashi, 1982) and Wieringa et al., (1984) and more recently Iwazumi and Tung introduced two (Iwazumi, 1982; Tung, 1986) very sensitive designs. None of these meets all the above design criteria. The design proposed by Fay and coworkers is very sensitive, and has a high resonant frequency and a good long term stability, but it has a rather limited force range and is very large in physical dimensions, owing to its cold light source and light conduction cables, which make it sensitive to mechanical noise. The Meiss type transducers and the design by Minns \& Franz, according to the literature, (Canaday \& Fay, 1976) lack the necessary stability and sensitivity. For the Meiss transducers this is probably owing to the spring or pivot suspension of a lever beam, whereas both designs do need considerable alterations to permit single cell attachment. The design by Ishii employs a very long light path, compromising long term stability, and uses suction as a method of cell attachment with a non axial pull on the cell. The design by Wieringa et al, (1984) involves an insufficient method of cell attachment showing slip at a force level of approximately $1.5 \mu \mathrm{N}$ (Van Dijk et al., 1984; Glerum \& Van Mastrigt, 1990) and has a poor bandwidth

\footnotetext{
* To whom all correspondence should be addressed at Department of Urology, Room EE 1630, Erasmus University, P.O. Box 1738, 3000 DR Rotterdam, The Netherlands.
} 
due to its video method for position detection. Also the maximum length to which the cell can be stretched is rather limited. In Iwazumi's design stretching of the cells is limited to a few micrometers. The transducer system by Tung also seems to be rather impractical as far as the application of length changes is concerned and lacks a truly longitudinal pull on the cell, making recurrent calculations of the cell's contact region with the probe tip necessary as its diameter changes with increasing length.

Therefore a new type of sensitive, rugged, but also small transducer system, fit for isometric contraction- and stress relaxation measurements, with a longitudinal, horizontal working axis, was developed.

\section{Principals of operation}

\section{Mechanical}

The system is principally formed by a stainless steel rod suspended at two points by quartz cantilever beams. The width $(1.0 \mathrm{~mm})$ of the quartz cantilever beams is large compared to their thickness $(80 \mu \mathrm{m})$, so that bending takes place in one preferred plane. As the rod is suspended at two, rather distant, points, no up-down or sideways movement or inclination can take place, rendering a system moving according to one horizontal axis.

As the bending of one beam is given by:

$$
d^{\prime}=4 F L^{3} /\left(E b h^{3}\right)
$$

where: $d^{\prime}=$ displacement $(\mathrm{m}), F=$ force $(\mathrm{N}), L=$ length of the beam (m), $\mathrm{E}=$ elastic modulus of quartz $\left(\mathrm{Nm}^{-2}\right)$, $b=$ width of the beam $(\mathrm{m})$, and $h=$ thickness of the beam (m), (McLauglin, 1977), the displacement of the rod due to bending of two quartz suspension beams is given by:

$$
d=2 F L^{3} /\left(E b h^{3}\right)
$$

where: $d=$ displacement of the rod $(\mathrm{m})$.

The compliance of the moving system thus is given by:

$$
C=d / F=2 L^{3} /\left(\mathrm{Ebh}^{3}\right)
$$

where: $C=$ compliance $(\mathrm{m} / \mathrm{N})$.

The resonant frequency is determined by:

$$
f=1 / 2 \pi \times \sqrt{1 /(C \times m)}
$$

where: $f=$ resonant frequency $(\mathrm{Hz}), m=$ moving mass (kg), (McLaughlin, 1977).

\section{Optical}

A vane, containing a tiny slit, is connected to the rear end of the rod and interrupts a homogeneous infra red light bundle. Displacement of the rod causes a small displacement of the resultant light spot projected on a dual planar photo diode pair. The difference in the photo voltages from both diodes forms a measure for the position of the light spot and thus for the position of the rod.

\section{Electrical}

A position dependent output signal is obtained by amplifying the difference signal from the photo diodes by a differential amplifier. Since both the sensitivity and the capacitance of the photo cells are dependent on the width of the $\mathrm{P}-\mathrm{N}$ border layer, an inverse bias voltage is applied to enhance cell sensitivity and to obtain a maximum cut off frequency.

To avoid DC drifting, resulting from the high gain input stages and, even more important, from infra red light emission of surrounding structures, a carrier system, involving a chopper phase detection technique is used. In this way $1 / f$ noise of the input stages is also converted to a frequency band above the low-pass frequency of the output filter, thus effectively increasing the signal to noise ratio.

\section{Construction details}

\section{Mechanical}

The transducer consists of 4 major mechanical parts, (Fig. 1A, B): (1) a main body, (2) a light path tube, (3) an active element suspension cylinder and (4) a motor driven mechanical zero adjustment mechanism.

The main body is formed by a brass cylinder with an external diameter of $40 \mathrm{~mm}$, and a length of $60 \mathrm{~mm}$. Its front end is open and has an inner diameter of $20 \mathrm{~mm}$, its rear end is closed and accommodates the light path tube through an $18 \mathrm{~mm}$ diameter cross hole, perpendicular to the main shaft. The light path tube is also a brass cylinder, into whose ends the infra red power LED and the bicellular photo cell, with their respective holders and fastening screws, exactly fit.

The active element suspension cylinder is machined from stainless steel, and fits like a brass-steel bearing into the main body. The rod runs through a $2 \mathrm{~mm}$ diameter longitudinal hole in the suspension cylinder. It is made of the same type of stainless steel, in order to minimise expansion differences between the rod and the suspension cylinder caused by temperature changes. At the rear end of the rod a stainless steel vane, with a $0.8 \mathrm{~mm}$ wide, $5 \mathrm{~mm}$ high vertical slit is point welded. The quartz suspension beams are glued to the front and back rims of the suspension cylinder, and to the rod, with ultra violet light bounding glue, (type: Loctite U.V. glue 358, Loctite Limited, Dublin, Ireland).

The front of the suspension cylinder is covered with a perspex cover through which the rod tip extends approximately $3 \mathrm{~mm}$, in order to prevent temperature differences between the two quartz suspension beams.

The suspension cylinder can be shifted in and out of the transducer main body and set to its mechanical zero position by a DC motor driven screw spindel.

Optical

The bi-cellular photo diode is soldered with its back to a small brass cylinder; both fit exactly in a mounting hole in 


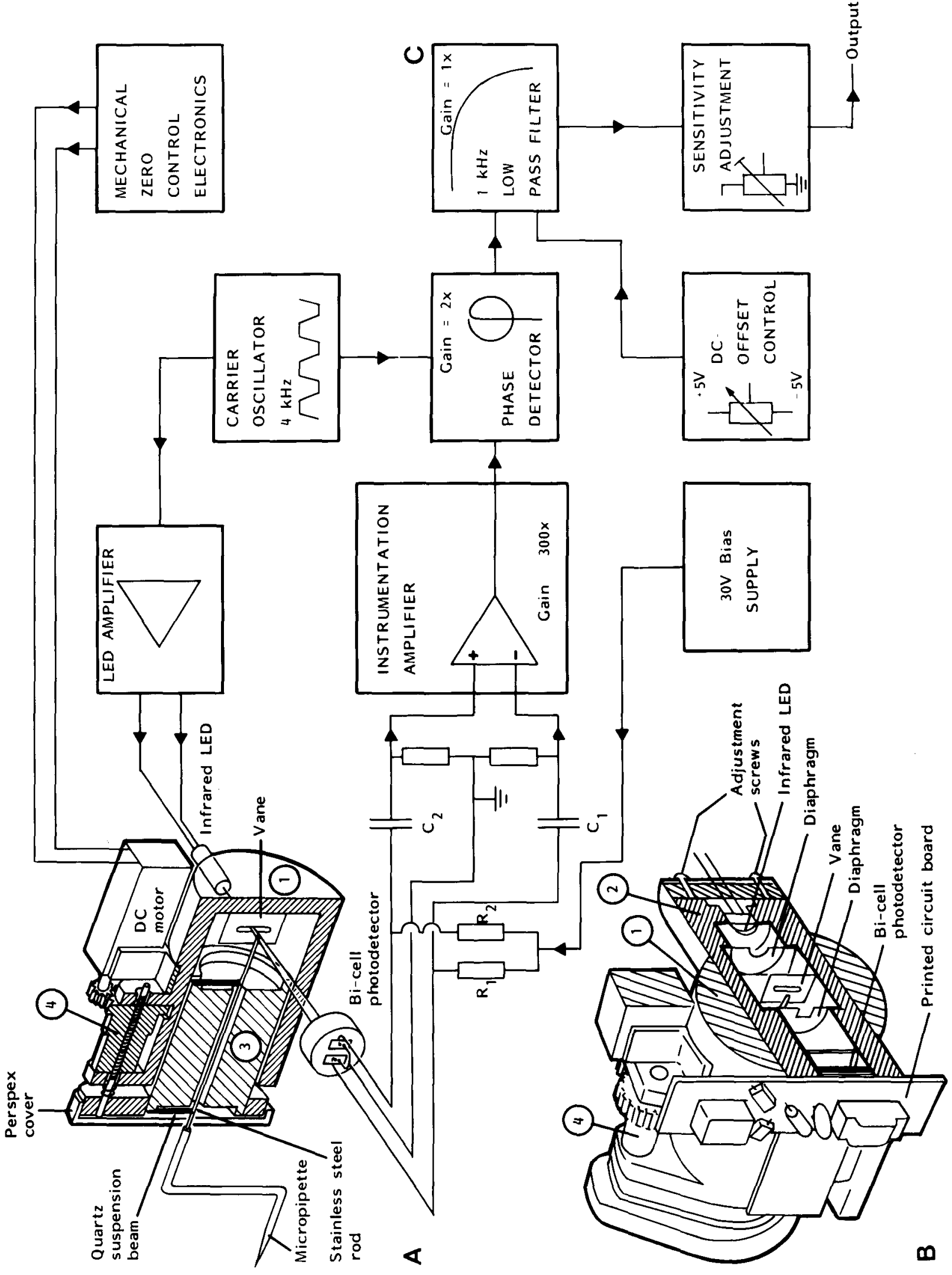

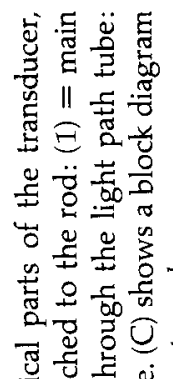

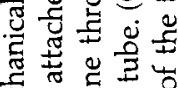

它

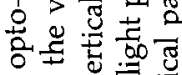

它运要

용.

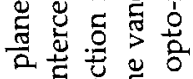

త].

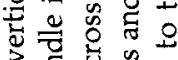

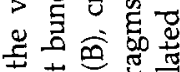

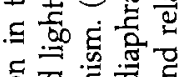

을

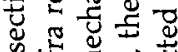

뜰

흘 동용

के

으워웡

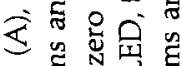

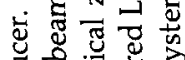

赵焉声

亏현

定 11 至

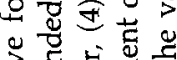

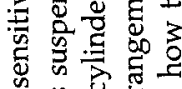

范它氙

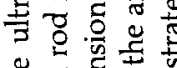

$\leftrightarrows \frac{\mathrm{q}}{5}$

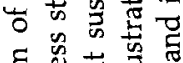

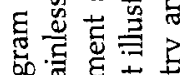

न के

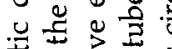

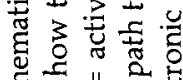

फू.

r 要

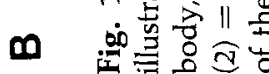


the light path tube. The back of this cylinder is soldered to a double sided glass-epoxy printed circuit board containing the primary amplifier stages. Three screws both align the photo cell and fix this combination to the light path tube.

The infra red power LED, also having a small printed circuit board at its back, is mounted in a similar way to the other end of the light path tube, but has three additional alignment screws to direct the light bundle exactly at the photo cells. To prevent undesirable stray light effects, two diaphragms are placed inside the light path tube, one in front of the LED and one in front of the bi-cell photo detector.

\section{Electrical}

Figure IC shows a block diagram of the electronics and their connections to the opto-mechanical parts of the transducer system.

Central to the design is a $4 \mathrm{kHz}$, square wave, $50 \%$ duty cycle, carrier oscillator which injects its signal both to the LED driver amplifier and to the chopper phase detector. The LED driver circuit sets the quiescent current of the LED and amplifies the carrier signal to the proper output power level.

The photo cells are supplied, through resistors 1 and 2, with a $30 \mathrm{~V}$ negative bias voltage to ensure optimum sensitivity, linearity and bandwidth.

The AC component of the photo voltages of both photo diodes is fed through capacitors 1 and 2 to the differential inputs of an instrumentation amplifier circuit with an overall differential gain of 300 .

The differential photo signal is demodulated in a chopper type phase detector circuit by switching an operational amplifier from non-inverting to inverting gain in phase with the carrier signal, resulting in a position dependent DC voltage.

An elliptical type, $1 \mathrm{kHz}$ low-pass output filter, removes all undesired high frequency signal components and provides optimum carrier frequency depression.

The DC offset control allows for accurate zeroing and subtraction of high passive force levels, using a ten turn precision potentiometer at a rate of $1 \mathrm{~V}$ per revolution to a maximum offset of $\pm 5 \mathrm{~V}$.

At the final output of the electronic circuit the overall transducer sensitivity can be calibrated to a desired level, e.g. $10 \mu \mathrm{N} \mathrm{V}^{-1}$.

A separate electronic system provides step-wise control of the DC motor which is driving the mechanical zero adjustment screw spindel, resulting in displacement steps of $0.2 \mathrm{um}$.

\section{Calibration and performance}

A prototype of the transducer was built using two quartz $\left(E=7 \times 10^{10} . \mathrm{N} \mathrm{m}^{-2}\right)$ suspension beams, each of $13.0 \mathrm{~mm}$ length, $1.0 \mathrm{~mm}$ width and $80 \mu \mathrm{m}$ thickness. As the glue droplets immobilized the suspension beams over a length of $0.5 \mathrm{~mm}$ at each end, the effective length of the beams was approximately $12.0 \mathrm{~mm}$. Equation (3) then predicts a compliance of $0.1 \mu \mathrm{m} \mu \mathrm{N}^{-1}$. Since the weight of the rod with the vane welded to it is approximately $60 \mathrm{mg}$, this will yield, according to equation (4), a resonant frequency of $64 \mathrm{~Hz}$. Adding the weight of the micro-pipette $(30 \mathrm{mg})$ yields a resonant frequency of approximately $50 \mathrm{~Hz}$.

To calibrate the transducer a force generator after Minns (Minns, 1971) was constructed, using a $100 \mu \mathrm{A}$ moving coil meter with middle zero position. To drive the force generator, a dedicated wave form- and DC level generator was developed, producing 5 preset frequencies, each with a triangular, sinus or square wave output, and a regulated $D C$ output, ranging in each case from 0 to $100 \mu \mathrm{N}$ or 0 to $1000 \mu \mathrm{N}$ effective force at the needle tip. This force generator was calibrated, up to $0.1 \mu \mathrm{N}$ accuracy using an electronic micro-balance, type Sartorius 2004 MP (Sartorius GMBH, Goettingen, West Germany), of the electronic null compensation type.

Calibration was performed with the transducer mounted on the microscope stage and a micro-pipette mounted to the transducer rod tip. The force generator was mounted on a modified Zeiss IM object stage movement mechanism, with DC motor controls, so as to function as a 3-D micromanipulator, to position the calibrator accurately. The tip of the moving coil meter needle was placed behind the vertical part of the Z-bend micro-pipette, so that a pulling force could be exerted on the transducer's rod. Displacement of the tip of the micropipette was monitored simultaneously through the microscope, thus checking proper function and compliance at higher force levels. Using the sensitivity adjustment circuitry the transducer was trimmed to an output of $\mathrm{IV}$ per $10 \mu \mathrm{N}$. Figure $2 \mathrm{~A}, \mathrm{~B}$ shows examples of the force signal resulting from calibration with a square wave and a triangular signal, Fig. 2A illustrates the pulse signal behaviour and the stability of the system at small forces, Fig. 2B illustrates the wide range linearity. Both signals were sampled with a PDP 11 type computer at 10 samples per second without any extra filtering or compensation networks. Table 1 . gives an overall resume of the mechanical and electrical properties of the transducer system which are in good comparison with the above calculated values.

The linear response of the transducer is limited to plus or minus $12 \mathrm{~V}$ by the dynamic range of the output filter. As the preceding circuitry shows a linear response between plus and minus $14 \mathrm{~V}$, the use of the DC-offset control allows a linear range of minus 140 to plus $140 \mu \mathrm{N}$.

\section{Discussion}

The design considerations set out at the beginning of this paper have almost completely been met at a very limited cost. A very rugged transducer has been constructed, with a large dynamic range, good linearity over this range, and 

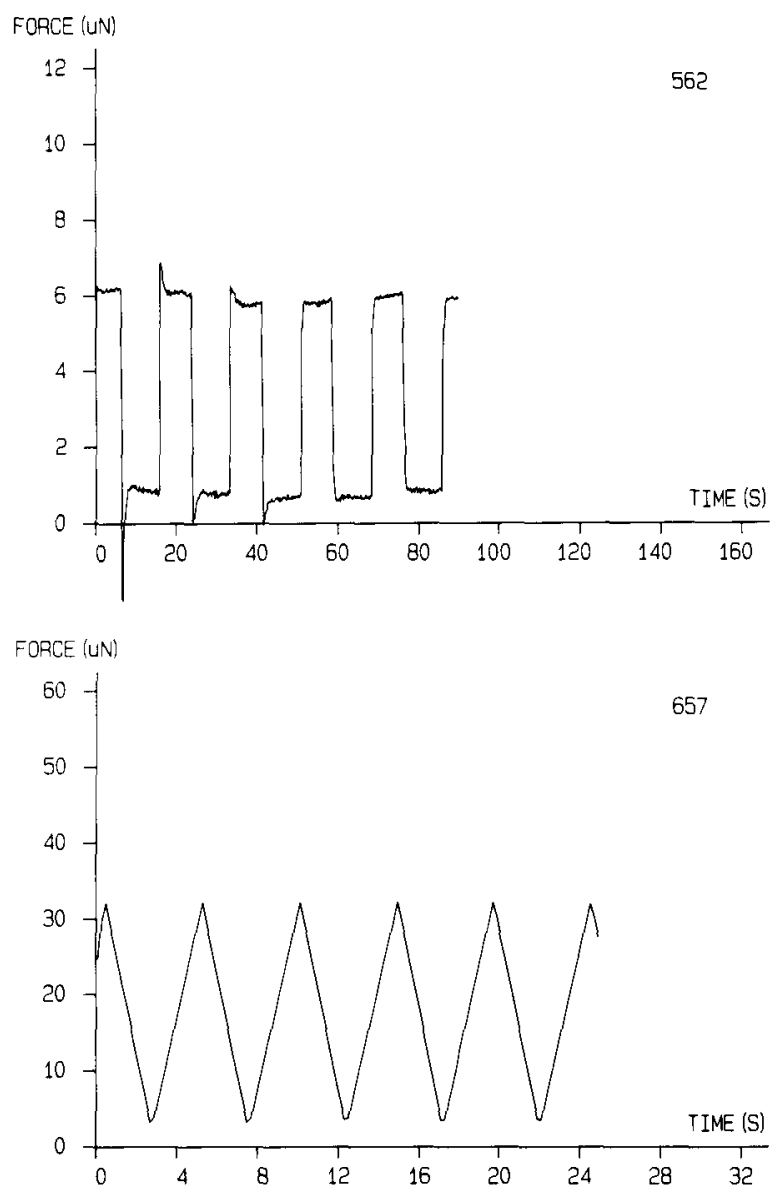

Fig. 2. (A) typical response of the force transducer to a square wave calibration force of $5 \mu \mathrm{N}$ p-p amplitude with a repetition frequency of $0.05 \mathrm{~Hz}$. The base line has been offset to approximately $1 \mu \mathrm{N}$ in order to show negative overshoot. The recording was made under measuring circumstances with a micro-pipette mounted to the transducer rod and its tip submerged in the cell incubator bath. The signal was sampled at $10 \mathrm{~Hz}$ without filtering. (B) typical response of the force transducer to a triangular calibration force of $30 \mu \mathrm{N} p-\mathrm{p}$ amplitude with a repetition frequency of $0.2 \mathrm{~Hz}$. The base line was offset to show clearly the lower parts of the signal. The recording was made under measuring circumstances with a micro-pipette mounted to the transducer rod and its tip submerged in the cell incubator bath. The signal was sampled at $10 \mathrm{~Hz}$ without filtering.

an acceptable sensitivity and signal to noise ratio. The costs of the electronic and mechanical parts of this transducer are in the order of $\$ 200.00$ and with respect to the most delicate part of the system, a number of spares can be manufactured in advance. The construction of the whole system does not require any special machinery or testing equipment, and takes approximately 1 manweek for the electronics and 1 manweek for the mechanical parts, including spare cylinders.

More specifically demands (1) to (4), (7), (8) and (10) to (14) have completely been met. Long term stability, (5), is in the order of $1-2 \mu \mathrm{Nh}^{-1}$, and is limited by temperature effects, (9), that influence the stability and position of
Table 1: Force transducer properties as measured under experimental conditions with the microscope illumination switched on and the object stage- and cell incubator heating at a working temperature of $37^{\circ} \mathrm{C}$, after $4 \mathrm{~h}$ of initial warm up in a acrylic sheet cabinet (unless otherwise specified). Electrical properties are expressed in $\mu \mathrm{N}$ for a transducer calibrated to a typical output signal of $100 \mathrm{mV} \mu \mathrm{N}^{-1}$.

\begin{tabular}{|c|c|}
\hline Sensitivity & $100 \mathrm{mV} \mu \mathrm{N}^{-1}$ \\
\hline Linear range & $\pm 120 \mu \mathrm{N}^{*}$ \\
\hline Maximum DC offset & $\pm 50 \mu \mathrm{N}$ \\
\hline \multicolumn{2}{|l|}{$\begin{array}{l}\text { Noise level: } \\
\text {-electrical, }\end{array}$} \\
\hline $\begin{array}{l}\text { power LED off } \\
\text { electrical, }\end{array}$ & $0.001 \mu \mathrm{N}$ p-p \\
\hline power LED on & $0.01 \mu \mathrm{N}$ p-p \\
\hline - total system & $<0.2 \mu \mathrm{N} \mathrm{p-p^{ \dagger }}$ \\
\hline Resolution & $<0.1 \mu \mathrm{N}^{+}$ \\
\hline \multicolumn{2}{|l|}{$\begin{array}{l}\text { Long-term drift: } \\
\text { electrical }\end{array}$} \\
\hline — total system & $0.3 \mu \mathrm{N} / \mathrm{sec}=\mathrm{I}-2 \mu \mathrm{N} \mathrm{h}^{-10}$ \\
\hline Compliance & $0.1 \mu \mathrm{m} \mu \mathrm{N}^{-1}$ \\
\hline Resonant frequency & $60 \mathrm{~Hz}$ without pipette \\
\hline Moving mass & $60 \mathrm{mg}$ without pipette \\
\hline
\end{tabular}

* without using DC offset capability. ${ }^{+}$depending on the mechanical noise conditions at the site of experimentation. $\$$ immediately after switch on. ${ }^{\circ}$ after 4 hours warming up at working conditions.

the cantilever-rod system. An important factor in temperature stability is formed by differences in the material properties (thermal expansion coefficients) of both rod and suspension cylinder. Another factor is the possible difference in temperature between both quartz suspension beams. This was controlled to a satisfactory level by adding a perspex shield to the front of the transducer. A final factor is formed by the temperature dependent properties of the glue, with which the quartz suspension beams are fastened; dual component epoxy types of glue proved to have worse temperature and elastic properties than the ultra violet light bounding glue that was finally used. To minimise these temperature effects, the whole measuring apparatus, including microscope and micro manipulator controls, was encased in an acrylic sheet cabinet.

In order to realise further improvement of thermal stability, all parts of the cantilever-rod system and suspension cylinder could be made from quartz, whereas the other parts of the transducer could be machined from glass ceramics such as Zerodur (Brehm \& Van Grootel, 1985), which has a temperature coefficient of almost zero in a temperature range of $-30^{\circ} \mathrm{C}$ to $+60^{\circ} \mathrm{C}$.

Sensitivity, ( 6$)$, is only limited by the average noise level, which is in our case set by the ambient mechanical noise level. Although the total measuring apparatus was mounted on a vibration isolating table at the quietest part of the university's premises, most of the noise level 
measured at the transducer's output was due to environmental mechanical vibrations. This was confirmed by interchanging the cantilever system with a solid metal dummy, which reduced the noise output to the level of the electronic's noise with the LED excitation power switched on (see Table 1).

Comparing this transducer design to other micro force transducers described in the literature, it is found to perform in most aspects equally as well as the transducer of Warshaw and Fay (1983) but is much smaller in physical dimensions, due to its electronically more sophisticated displacement detection system. Also the maximum linear range is considerably larger, as the detection of displacement is not limited to the width of the grating in the Ronchi rulings as in Warshaw's transducer, but limited to the width of the photo diodes which are each $3.0 \mathrm{~mm}$ wide, resulting in a theoretical displacement range of plus and minus $1.5 \mathrm{~mm}$. As high gain is needed to obtain sufficient sensitivity, already at the first amplifier stage maximum range is limited electronically. If the rod mass were equally low, the frequency response of both transducers would also be comparable. The present fairly low resonant frequency resulted from the use of rather long and heavy micro-pipettes, bent in a $Z$ like fashion, so as to overcome differences in height and distance from the transducer to the position of the cell, which in its turn, to maintain mechanical stability, necessitated the use of a rod of considerable length and mass. Improving this would require major changes in the arrangement of the cell incubator and surrounding instruments.

Comparing the transducer with the series of transducers reported on by Meiss (1974), it is found to be by far better suited for single cell measurements, as it is more sensitive and shows better long term stability. Also an equivalent assortment of sensitivity or force ranges can be achieved, by exchanging the suspension cylinder for a type of cylinder containing thicker, less compliant, suspension beams. The same holds for the design of Minns \& Franz (1972). Compared to the design by Ishii (1982, 1988), our transducer does not need recalibration before or after each experiment, as the compliant part of it remains the same for every following experiment. Also there is no need for exact optical alignment of the cell preparation in the position detection field and long optical pathways are avoided, whereas influences from external heat or light sources are eliminated by using the synchronous pulsed light of the carrier system instead of a constant light- or laser beam.

Considering the design of Wieringa et al., (1984), the new transducer is found superior in frequency response and in the amplitude of stretch that can be applied to a smooth muscle cell.

In contrast to Iwazumi's design (1982), no currents or magnetic fields, possibly interfering with electro-physiological measurements, are conducted or induced within the experimentation bath, whereas compared to both the former and Tung's transducer (Tung, 1986) extended length changes are much more easily performed. With respect to these last two designs it should be considered that both transducers are extremely sensitive and possibly intended for another type and level of single cell measurements.

It is concluded that the described transducer is very suitable for measuring length-tension relationships and the length dependence of force development. Such measurements were performed on urinary bladder and uterine smooth muscle cells. The results are reported in part II and III of this publication.

Throughout a period of 6 months the transducer performed according to specifications, without any recalibration procedures being necessary, except for the only occasion a suspension cylinder had to be changed due to accidental mechanical damage.

\section{Acknowledgements}

The authors wish to thank C. J. B. Schellevis of the mechanical workshop of the Central Research Workshops of the Erasmus University in Rotterdam for machining the first prototype of the transducer. We also thank $F$. Schumacher and A. A. Brouwer from the same department for the many alterations that were skilfully realized. We are much obliged to J. B. F. Ekas of the glass technical workshop of the Central Research Workshops for providing us with several types of quartz suspension beams. J. V. de Bakker and C. J. Keemink from the department of Biomedical Physics and Technology were very helpful in discussing the pitfalls of practical electronic engineering, and C. Goedegebuur from the same department taught us the many skills necessary to machine numerous bits and pieces of the transducer and of the remaining smooth muscle cell measurement apparatus.

\section{References}

BREHM, R. \& GROOTEL, P. VAN (1985) Toepassing van glaskeramiek in een precisie-meetapparaat. Nederlandse Vereniging voor Glastechniek informatie, 2, 2-3.

CANADAY, P.G. \& FAY, F.S. (1976) An ultrasensitive isometric force transducer for single smooth muscle cell mechanics. J. Appl. Physiol. 40, 243-6.

DIJK, A. M. VAN, WIERINGA, P. A., MEER, M. VAN DER \& LAIRD, J. D. (1984) Mechanics of resting isolated single vascular smooth muscle cells from bovine coronairy artery. Am. J. Physiol. 246, C277-87.

GLERUM, J. J., MASTRIGT, R. VAN, (1990) Mechanical properties of mammalian single smooth muscle cells, II: Evaluation of a modified technique for attachment of cells to the measurement apparatus. J. Musc. Res. Cell Motil. 11, $338-343$.

ISHII, N. \& TAKAHASHI, K. (1982) Length-tension relation of single smooth muscle cells isolated from the pedal retractor muscle of Mytilus edulis. J. Musc. Res. Cell Mot. 3, 25-38.

ISHII, N., SIMPSON, A. W. M. \& ASHLEY, C. C. (1988) Intracellular free calcium $\left(\left[\mathrm{Ca}^{2+}\right]_{\mathrm{i}}\right)$ and the 'catch' contraction in isolated molluscan smooth-muscle (ABRM) cells. (abstract) J. Musc. Res. Cell Mot. 9, 463. 
IWAZUMI, T. (1982) High performance instrument for myofibrillar mechanics. (abstract) Bioph. J. 37, 357a.

McLAUGHLIN, R. J. (1977) Systematic design of cantilever beams for muscle research. J. Appl. Physiol. 42, 786-94.

MEISS, R. A. (1971) An isometric muscle force transducer. J. Appl. Physiol. 30, 158-60.

MEISS, R. A. (1974) A versatile transducer system for mechanical studies of muscle. J. Appl. Physiol. 37, 459-63.

MINNS, H. G. (1971) A voltage-controlled force generator for calibrating sensitive transducers. J. Appl. Physiol. 30, 895-6.
MINNS, H. G. \& FRANZ, G. N. (1972) A low-drift transducer for small forces. J. Appl. Physiol. 33, 529-31.

TUNG, L. (1986) An ultrasensitive transducer for measurement of isometric contractile force from single heart cells. Pflügers Arch. 407, 109-15.

WARSHAW, D. M. \& FAY, F. S. (1983) Cross-bridge elasticity in single smooth muscle cells. J. Gen. Physiol. 82, 157-99.

WIERINGA, P. A., MEER, M. VAN DER, DIJK, A. M. VAN \& LAIRD, J. D. (1984) Sensitive force transducer system for mechanical studies of single isolated vascular smooth muscle cells. Med. \& Biol. Eng. \& Comp. 22, 130-7. 